\title{
Peritoneal Tuberculosis Presenting as Chronic Ascites With Scrofula: A Case Report
}

\begin{abstract}
Peritoneal tuberculosis (PTB) is a common type of extrapulmonary tuberculosis; however, due to variety of clinical presentations, diagnostic challenges do occur. The nonspecificfeatures of this disease can lead to diagnostic delays and the development of complications. In addition, PTB can mimic a malignancy, especially in women who present with ascites and elevated cancer antigen (CA) 125 levels. A high index of suspicion is an important factor in an early diagnosis. Moreover, an early diagnosis and the initiation of antituberculous therapy are essential for preventing morbidity and mortality. Fortunately, most of these patients respond very well to standard antituberculous therapy.

Here, we have reported the case of a young female patient who presented with chronic ascites, mild abdominal tenderness, and later, scrofula. Ultimately, she was diagnosed with PTB based on her test results. We expect that this case report will contribute to the existing literature on this subject.

Keywords: Chronic Ascitis; Extrapulmonary Tuberculosis; Ovarian Neoplasms; Peritonitis, Tuberculous; Tuberculosis, Lymph Node

CMI 2019; 13(1): 29-34

http://dx.doi.org/10.7175/cmi.v13i1.1416
\end{abstract}

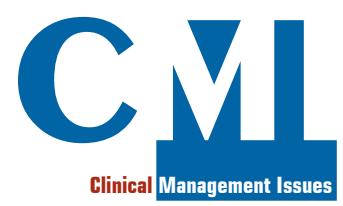

Case Report
Department of Internal Medicine, Pietersburg Provincial Hospital, Limpopo University, Polokwane, South Africa

\section{INTRODUCTION}

Despite the availability of effective treatments and the use of live attenuated vaccines in many parts of the world, tuberculosis (TB) remains one of the deadliest communicable diseases. Peritoneal TB (PTB) is seen in $4.9 \%$ of all extrapulmonary TB (ETB) cases [1]. Although TB affects the lungs in the majority of patients, ETB is the initial presentation in approximately $20 \%$ of adults, and it primarily involves the lymph nodes, pleura, and abdomen. ETB occurs more commonly in immunosuppressed individuals and young children. In those individuals with the human immunodeficiency virus (HIV), it occurs in more than $50 \%$ of the cases [1]. Although the Republic of South Africa has made notable progress in reduc-
Why Do We Describe This Case

Tuberculosis (TB) has become a global problem with the increasing number of immunocompromised patients. This disease is also spread by overcrowding, poverty, and drug resistance. The abdominal $T B$ rates are rising, and the nonspecific features of this type of extrapulmonary TB create difficulties in establishing a diagnosis. However, the prompt initiation of treatment can help prevent morbidity and mortality. This article should encourage clinicians to consider the possibility of abdominal $T B$ in the correct clinical setting in order to ensure a timely diagnosis and appropriate treatment
Corresponding author Galina Bogoslovskaya albogoslovsky@gmail.com

Received: 27 February 2019 Accepted: 20 May 2019 Published: 14 June 2019 


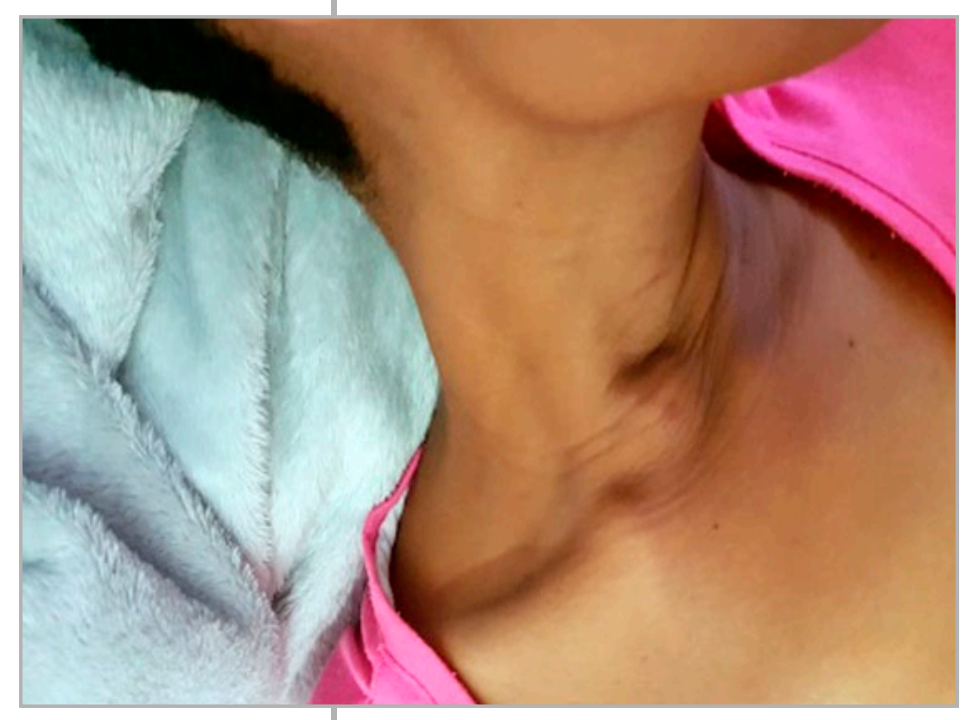

Figure 1. Single diffuse swollen area of approximately $4 \times 3 \mathrm{~cm}$ with ill-defined borders in the right cervical region.

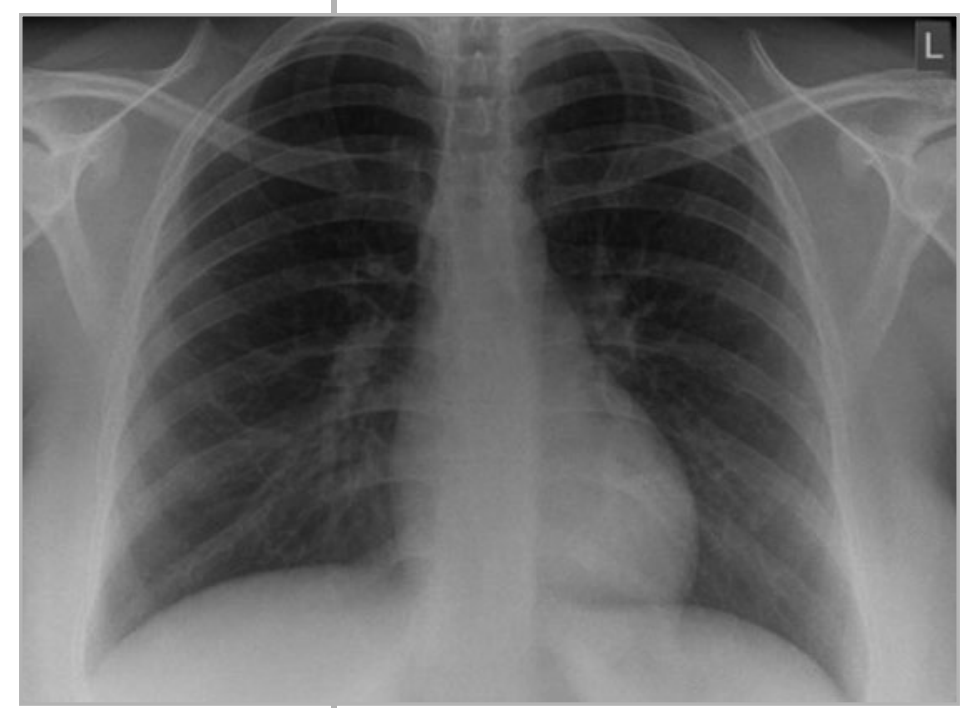

Figure 2. Chest radiographs showing no pathological signs.

ing the TB prevalence and deaths due to TB, as well as improving the treatment outcomes for new sputum smear-positive TB cases, the TB burden is still enormous [2,3].

Ovarian cancer is one of the most difficult gynecological cancer types to diagnose and tenderness in the right side of her neck, and she felt a small lymph node in the cervical region. At the same time, she began to exhibit a low-grade fever and sweating. Based on the above mentioned background information, she was referred to the Pietersburg Provincial Hospital Internal Medicine Department (South Africa) for a diagnostic review and admission.

Upon examination, this patient appeared ill but afebrile, with no acute respiratory distress (respiratory rate of 18) or unstable vital signs. A visual inspection showed a single diffuse swollen area of approximately $4 \times 3 \mathrm{~cm}$ with ill-defined borders in the right cervical region. The overlying skin was the same color as the surrounding skin. Upon palpation, the mass was slightly tender, fluctuant, compressible, mobile, and showed signs of matting (Figure 1).

However, her other lymph nodes were not palpable, her lungs were clear, and her cardiovascular examination was normal. Her markedly distended abdomen was notable for massive ascites with no signs of peritonitis. There was mild tenderness in the abdomen upon palpation. Moreover, there was no lower extremity swelling. The remainder of the exam was significant for an unhealed 
low laparotomy scar that was oozing a yellow-colored fluid. There were no stigmata of chronic liver disease or a hernia. She had mild anemia, with a hemoglobin level of 11 $\mathrm{g} / \mathrm{dL}$ and a normal white blood cell count $\left(7.300 \times 10^{9} / \mathrm{L}\right)$ and her blood biochemical profile was normal. In addition, her viral hepatitis test and HIV profile were negative.

Chest radiographs findings did not indicate pulmonary tuberculosis (Figure 2). The sputum was negative $\left(\mathrm{GeneXpert}{ }^{\circledR} ; \mathrm{Ce}-\right.$ pheid, Sunnyvale, CA, USA). Her abdominal and thoracic ultrasound revealed massive ascites with mild septation.

At the time of presentation, this patient was taking furosemide $40 \mathrm{mg}$ daily and spironolactone $25 \mathrm{mg}$ daily, which were prescribed by her GP one month before her admission. We stopped these medications and admitted the patient to the medical ward. A puncture of the cervical mass resulted in the aspiration of $15 \mathrm{ml}$ of yellow turbid liquid with marked caseous necrosis. Laparocentesis revealed a yellow-color translucent fluid.

The ascites fluid had a high protein content, low glucose level, and marked lymphocytes with a serum ascites albumin gradient (SAAG) lower than 1.1 excluding portal hypertension. Furthermore, the ascites fluid adenosine deaminase (ADA) levels were markedly high (45 U/L; the normal limit is $<30 \mathrm{U} / \mathrm{L}$ ).

The histological examination of the peritoneal biopsy revealed numerous caseating epithelioid granulomas with multinucleated Langhans giant cells lymphocytes, and focal caseation. Special staining, such as the Ziehl-Neelsen stain, identified occasional pathological acid-fast bacillus organisms that were consistent with TB. Therefore, the ovarian malignancy diagnosis was ruled out, and PTB with tuberculous lymphadenitis was established. Afterwards, patient was prescribed the antituberculous treatment Rifafour ${ }^{\circledR} 3$ tablets (each tablet contains: rifampicin $150 \mathrm{mg}$, isoniazid $75 \mathrm{mg}$, pyrazinamide $400 \mathrm{mg}$, and ethambutol $275 \mathrm{mg}$ ) [5].Two days after the antituberculous treatment began, the general condition of this patient improved significantly, and she was discharged from the hospital 10 days after her admission to the medical ward. A careful retrospective evaluation of her respiratory system showed no evidence of pulmonary TB. However, the later results of her ascitic fluid culture were positive for Mycobacterium tuberculosis. The mycobacterial culture of the lymph node aspirate showed negative result.

\section{DISCUSSION}

PTB can be acute or chronic, and traditionally, it has been divided into four types:

1. the wet ascitic type is more common, and it is associated with a large amount of free or loculated fluid in abdomen. The ascitic fluid usually exhibits a high density due to the increased protein content of the inflammatory exudate. In addition, associated peritoneal enhancement is usually present;

2. the dry, fixed, fibrotic type of PTB includes the involvement of the omentum and mesentery, and it is characterized by presence of matted bowel loops upon imaging. This type of PTB exhibits a fibrous peritoneal reaction, peritoneal nodules, and the presence of adhesions;

3. occasionally, loculated encysted ascites can be present, which constitutes the third type of PTB [6,7]. Abdominal cocoon formation is an uncommon manifestation of fibrotic type of PTB that is characterized by development of fibrous membrane-like sac around of small intestine loops. The clinical presentations included predominantly abdominal pain and intestine obstruction [8];

4. finally, the purulent and rare form of PTB usually develops secondarily to tuberculous salpingitis, with the pockets adherent to the intestine and omentum containing TB pus ("cold” abscesses).

However, a combination of these features is usually noted [6].

PTB exhibits a diverse and nonspecific symptomatology, and no single test is adequate for this diagnosis in all patients [7]. Liver cirrhosis, chronic ambulatory peritoneal dialysis, diabetes mellitus, and HIV are all risk factors for PTB. However, abdominal TB in a non-HIV patient is still a challenging diagnosis requiring a high index of clinical suspicion. In this particular case, the postpartum condition could have been a risk factor for the development of $\mathrm{ETB}$, specifically $\mathrm{PTB}$ with scrofula [9-11].

The extrapulmonary forms of TB constitute approximately one-sixth of all cases, and the prevalence of ETB seems to be rising, particularly due to the increasing prevalence of acquired immunodeficiency syndrome. The abdomen is involved in $12 \%$ of the patients with ETB, and gastrointestinal involvement is found in $66 \%$ to $75 \%$ 
of the abdominal cases, with the terminal ileum and ileocecal regions being the most common sites of involvement. This frequency is higher, and it could double or even triple, in HIV-positive subjects. ETB can affect all age groups, with a predilection for women between 20 and 50 years of age [9].The peritoneal transplantation of $M . t u-$ berculosis is hematogenous, and it is mainly transplanted from primary infections that often go unnoticed. More rarely, it can be transplanted after a first gastrointestinal infection. In other cases, the peritoneal infection represents seeding from the abdominal lymph nodes [12].

Clinically, PTB can mimic advanced ovarian cancer, with pelvic pain, abdominal distension, weight loss, and palpation of an abdominopelvic mass being present in both conditions. However, the search for and absence of other signs, such as menstrual disorders ( $55 \%$ of the cases) and infertility (5\% to $10 \%$ of the cases), can be useful for the diagnosis of abdominal TB [13]. An association with other localizations, especially pulmonary, should be investigated, but its absence does not eliminate the diagnosis of PTB, as in our case. Several imaging techniques, such as ultrasonography and computed tomography, can sometimes guide the diagnosis. The existence of ascites with septas, peritoneal thickening, and heterogeneous enhancement are typical for inflammatory processes like TB. In addition, the CA125 level, which is an ovarian cancer biomarker of epithelial origin, can be high in several benign gynecological diseases (endometriosis, uterine fibroids, and pelvic inflammatory processes), extragynecological diseases (peritonitis, pancreatitis, hepatitis, nephrotic syndrome, and PTB), and nongynecological cancers with peritoneal metastases. In PTB cases, very high CA125 values (>1,000 U/ $\mathrm{mL}$ ) can be seen [14]. Moreover, $90.1 \%$ of PTB patient have elevated plasma CA125 levels with a mean value of $565 \mathrm{U} / \mathrm{mL}$ [13]. Therefore, CA125 has no place in the differential diagnosis between ovarian cancer and PTB. However, a decrease in the CA125 level has been correlated with the TB treatment response, and it has been indicated as a surveillance marker in antituberculous therapy [14].

Ascitis may be the first manifestation of a systemic disease or an otherwise unsuspected abdominal disease. In most cases, ascitis appears as part of a well-recognized illness, e.g. cirrhosis, congestive heart failure, nephrosis, or disseminated carcinomatosis. Occasionally, it may develop as an isolated finding in the absence of a clinically evident disease. In such cases, a thorough analysis of the ascitic fluid helps identify the etiology [11]. For instance, a SAAG value of $>$ or $<1$ $\mathrm{g} / \mathrm{dL}$ can differentiate between a transudate and an exudate fluid. A SAAG value of $>1$ is usually related to uncomplicated cirrhosis, alcoholic hepatitis, congestive heart failure, and Budd-Chiari syndrome, while a SAAG value of $<1$ is related to other causes, such as peritoneal carcinomatosis, tuberculous peritonitis, pancreatitis, serositis, pyogenic peritonitis, and nephritic syndrome. A certain diagnosis via an ascitic fluid analysis is made only after the detection of M. tuberculosis using either a direct examination or after a Lowenstein-Jensen medium culture. The ascitic fluid ADA ratio was also higher in patients with peritoneal tuberculosis than with other causes of ascites. ADA is useful diagnostic test for establishment of tuberculosis [15]. At present, an ascites ADA activity of $30 \mathrm{U} / \mathrm{L}$ is generally accepted as the cut-off level expected to yield the best results $[16,17]$. Tissue $\mathrm{Xpert}^{\mathrm{TM}} \mathrm{MTB} / \mathrm{Rif}$ is of limited use in diagnosis of peritoneal TB [18]. The other laboratory tests results are nonspecific, including mild anemia, inflammatory biomarker elevations, and an intradermal reaction to tuberculin [19]. However, serum $\mathrm{C}$ reactive protein $(\mathrm{CRP})$ is a very useful tool in the assessment of response to anti-tubercular therapy in abdominal tuberculosis [20].

In our case, the anatomopathological examination after the caseous granulation biopsy was sufficient to confirm the diagnosis. In addition, the search for mycobacteria using polymerase chain reaction can be useful for the diagnosis, with a sensitivity of $75-80 \%$ and a specificity of $85-95 \%$, but this technique is often unavailable in South African hospitals. As in the case of our patient, surgical exploration is necessary to confirm or rule out the suspicion of a malignant ovarian tumor. The first route can consist of either a laparotomy or laparoscopy. However, transvaginal or transabdominal ultrasound-guided biopsies can be used in cases with a strong suspicion of TB, thus limiting postoperative complications $[19,21]$. The histological study of biopsies makes it possible to correct the diagnosis by showing gigantocellular granulomas with caseous necrosis specific for Koch's bacillus. The treatment of PTB is essen- 
tially medical, and it is based on the daily administration of a quadritherapy combining isoniazid, rifampicin, ethambutol, and pyrazinamide for two months. Then, a four-month maintenance treatment is begun, using daily dual therapy combining isoniazid and rifampicin [5]. Steroids may be of benefit in peritonitis, but the evidence is scant and the routine use of steroids cannot be recommended [22].

A surgical procedure is especially indicated in cases with a compressive, obstructive or fistulised mass in order to flatten the caseous cavities [5,23].

Overall, PTB prevention is based on a $\mathrm{Ba}-$ cillus Calmette-Guérin vaccination, the detection of asymptomatic and atypical forms, chemoprophylaxis in the case of contact, and improvements in the hygiene of populations at risk [23].

\section{CONCLUSION}

Here we reported a case of postpartum PTB mimicking a malignant tumor. A 27-year-old woman who had a normal vaginal delivery six months previously presented to our department with ascites and an elevated serum CA125 level. PTB was diagnosed via a peritoneal biopsy. After undergoing treatment using the four-drug anti-TB regimen including isoniazid, rifampicin, ethambutol, and pyrazinamide, the patient recovered with the disappearance of ascites and a normal CA125 serum level after six months. These findings may lead to a misdiagnosis of ovarian cancer. This case emphasizes the fact that tuberculosis peritonitis should be considered as a differential diagnosis of ovarian cancer metastasis in patients with elevated serum CA125 values.

\section{Key Points}

- Tuberculosis (TB) can spread from the lungs and lymph nodes through the bloodstream to many sites, including the abdomen, particularly the peritoneum

- The TB symptoms depend on the affected organ, but they may be very atypical and nonspecific

- CA125, an ovarian cancer biomarker, can be elevated in PTB cases

- The diagnosis of PTB is based on the identification of $M$. tuberculosis in the infected fluid or a microscopic examination of the peritoneal tissue and culture

- A decrease in the CA125 level is correlated with the TB treatment response

- In patients with peritoneal tuberculosis the ascitic fluid and serum ADA activity was significantly higher than for other types of ascites

- Serum CRP remains very useful tool in the assessment of response to anti-tubercular therapy in abdominal tuberculosis

\section{Funding}

This article has been published without the support of sponsors.

Conflicts of Interest

The authors declare they have not competing financial interests concerning the topics of this article.

\section{REFERENCES}

1. Peto HM, Pratt RH, Harrington TA, et al. Epidemiology of extrapulmonary tuberculosis in the United States, 1993-2006. Clin Infect Dis 2009; 49: 1350-7; https://doi.org/10.1086/605559

2. Churchyard GJ, Mametja LD, Mvusi, et al. Tuberculosis control in South Africa: successes, challenges and recommendations. South Africa Med J 2014; 104 (3 Suppl 1): 244-8; https:// doi.org/10.7196/SAMJ.7689

3. Padayatchi N, Daftary A, Naidu N, et al. Tuberculosis: treatment failure, or failure to treat? Lessons from India and South Africa. BMJ Glob Health 2019; 4: e001097; https://doi. org/10.1136/bmjgh-2018-001097

4. Oge T, Ozalp S, Yalcin OT, et al. Peritoneal tuberculosis mimicking ovarian cancer. Eur J Obstet Gynecol Reprod Biol 2012; 162: 105-8; https://doi.org/10.1016/j.ejogrb.2012.02.010

5. Department Health, Republic of South Africa. National Tuberculosis Management Guidelines 2014. Available at http://www.tbonline.info/media/uploads/documents/national_tuberculosis_ management_guidelines_\%282014\%29.pdf (last accessed June 2019) 
6. Sood R, Sethu Madhavan M. Diagnostic approach to abdominal tuberculosis. In: Agarwal AK, Jain DG, editors. Clinical Medicine: A Practical manual for students and practitioners. Delhi (India): Jaypee Brothers Medical Publishers (P) Ltd, 2007; p. 249

7. Sanai FM, Bzeizi KI. Systematic review: tuberculous peritonitis-presenting features, diagnostic strategies and treatment, Alimentary Pharmacology and Therapeutics. Aliment Pharmacol Ther 2005; 22: 685-700; https://doi.org/10.1111/j.1365-2036.2005.02645.x

8. Sharma V, Singh H, Mandavdhare HS. Tubercular Abdominal Cocoon: Systematic Review of an Uncommon Form of Tuberculosis. Surg Infect (Larchmit) 2017; 18: 736-41; https://doi. org/10.1089/sur.2017.110

9. Bonura C, Carlo PD, Spicola D, et al. Rapidly growing mycobacteria in TB/HIV co-infection: a report of two cases focusing on difficulties in diagnosis and management. New Microbiol 2012; 35: 239-43

10. Shu CC, Wu VC, Yang FJ, et al. Predictors and prevalence of latent tuberculosis infection in patients receiving long-term hemodialysis and peritoneal dialysis. PLoS One 2012; 7: e42592; https://doi.org/10.1371/journal.pone.0042592

11. Huang LL, Xia HH, Zhu S. Ascitic Fluid Analysis in the Differential Diagnosis of Ascites: Focus on Cirrhotic Ascites. Clin Transl Hepatology 2014; 2: 58-64; https://doi.org/10.14218/ JCTH.2013.00010

12. Ramirez-Lapausa M, Menendez-Saldana A, Noguerado-Asensio A. Extrapulmonary tuberculosis: an overview. Rev Esp Sanid Penit 2015; 17: 3-11; https://doi.org/10.4321/S157506202015000100002

13. Daaloul W, Gharbi H, Ouerdiane N, et al. Disseminated peritoneal tuberculosis simulating ovarian cancer. Tunisia Med 2012; 90: 333-5

14. Kubota M, Suzuki J, Morisawa Y. Tuberculosis Peritonitis Mimicking Ovarian Cancer Metastasis. Intern Med 2019; 58: 763-4; https://doi.org/10.2169/internalmedicine.0752-17

15. Tao L, Ning HJ, Nie HM, et al. Diagnostic value of adenosine deaminase in ascites for tuberculosis ascites: a meta-analysis. Diagn Microbiol Infect Dis 2014; 79: 102-7; https://doi. org/10.1016/j.diagmicrobio.2013.12.010

16. Sharma SK, Ryan H, Khaparde S, et al. Index-TB Guidelines: Guidelines on extrapulmonary tuberculosis for India. Indian J Med Res 2017; 145: 448-63

17. Barua R, Hossain MA. Adenosine Deaminase in Diagnosis of Tuberculosis: A Review. Anwer Khan Modern Medical College Journal 2014; 5: 43-8

18. Bera C,Michael JS, Burad D, et al.Tissue Xpert ${ }^{\mathrm{TM}} \mathrm{MTB} /$ Rif assay is of limited use in diagnosing peritoneal tuberculosis in patients with exudative ascites. Indian J Gastroenterol 2015; 34: 3958; https://doi.org/10.1007/s12664-015-0599-7

19. Daley BJ. How is tuberculosis peritonitis (TP) diagnosed? Updated: Jan 11, 2017. Medscape. Available at https://www.medscape.com/answers/180234-55868/how-is-tuberculosisperitonitis-tp-diagnosed (last accessed June 2019)

20. Sharma V, Mandavdhare HS, Lamoria S, et al. Serial C-reactive protein measurements in patients treated for suspected abdominal tuberculosis. Dig Liver Dis 2018; 50: 559-62; https:// doi.org/10.1016/j.dld.2017.12.008

21. Vardareli E, Kebapci M, Saricam T, et al. Tuberculous peritonitis of the wet ascitic type: clinical features and diagnostic value of image-guided peritoneal biopsy. M Dig Liver Dis 2004; 36: 199-204; https://doi.org/10.1016/j.dld.2003.10.016

22. Soni H, Bellam BL, Rao RK, et al. Use of steroids for abdominal tuberculosis: a systematic review and meta-analysis. Infection 2019; 47: 387-94; https://doi.org/10.1007/s15010-018-1235-0

23. Tierney D, Nardell EA. Extrapulmonary Tuberculosis (TB). MSD Manual Professional version 2019. Last full review/revision April 2018. Available at https://www.msdmanuals. $\mathrm{com} / \mathrm{professional/infectious-diseases/mycobacteria/extrapulmonary-tuberculosis-}$ tb?query=abdominal\%20tuberculosis (last accessed June 2019) 